\title{
The Gumbel test and jumps in the volatility process
}

\author{
Christian Palmes and Jeannette H.C. Woerner
}

Preprint 2013-15

Dezember 2013

Fakultät für Mathematik

Technische Universität Dortmund

Vogelpothsweg 87

44227 Dortmund

tu-dortmund.de/MathPreprints 



\title{
The Gumbel test and jumps in the volatility process
}

\author{
Christian Palmes and Jeannette H.C. Woerner*
}

November 29, 2013

\begin{abstract}
In the framework of jump detection in stochastic volatility models the Gumbel test based on extreme value theory has recently been introduced. Compared to other jump tests it possesses the advantages that the direction and location of jumps may also be detected. Furthermore, compared to the Barndorff-Nielsen and Shephard test based on bipower variation the Gumbel test possesses a larger power. However, so far one assumption was that the volatility process is Hölder continuous, though there is empirical evidence for jumps in the volatility as well. In this paper we derive that the Gumbel test still works under the setting of finitely many jumps not exceeding a certain size. Furthermore, we show that the given bound on the jump size is sharp.
\end{abstract}

MSC 2010: 62G10, 62P05, 62G32

Keywords: jump test, stochastic volatility model, volatility process with jumps, Gumbel distribution, extreme value theory, high-frequency data

\section{Introduction}

In the last years the detection of jumps in stochastic volatility models based on high-frequency data has attained much attention, since this is an important task for modeling, risk assessment and statistical inference of the integrated volatility or volatility process itself, cf. [1], [3]. Recently Lee and Mykland [7] and Palmes and Woerner [9] proposed the Gumbel test, i.e. a test relying on extreme value theory, namely that a certain test statistics based on the maximum of increments of the log-price process converges to the Gumbel distribution under the null hypothesis of no additive jump component and to infinity otherwise. Palmes and Woerner [9] derived that this test compared to other tests based on power and multipower variation has the advantages that both the direction and the location of the jumps may be inferred. Furthermore, compared to the Barndorff-Nielsen and Shepard test based on bipower variation the Gumbel test possesses a larger power. However, so far it has only been shown that the Gumbel test works under the assumption of a continuous volatility process, though there is some empirical evidence that also volatility processes might possess jumps, cf. Jacod and Todorov [6]. Concerning the volatility process Lee and Mykland [7] need the assumption that it is $\alpha$-Hölder continuous for any $\alpha<1 / 2$, whereas Palmes and Woerner [9] relaxed the assumption to a general pathwise Hölder-continuous volatility process.

In this paper we show that the Gumbel test still works for a volatility process with finitely many jumps not exceeding a certain bound depending on the minimal attained volatility. Furthermore, we show that the derived bound on the jump size is sharp.

The outline of the paper is the following, in the next section we provide the necessary definitions and notation. In section 3 we prove the convergence of our test statistics under the new assumptions on the

\footnotetext{
*Lehrstuhl IV, Fakultät für Mathematik, Technische Universität Dortmund, D-44221 Dortmund, Germany, christian.palmes@math.tu-dortmund.de, jeannette.woerner@math.tu-dortmund.de
} 
volatility process and show that the bound on the size of the jumps in the volatility process is sharp. In section 4 we provide a small simulation study.

\section{Definitions and Notation}

Let $\left(\Omega, \mathcal{F},\left(\mathcal{F}_{t}\right)_{t \in[0,1]}, P\right)$ be a filtered probability space, which we assume to fulfill the usual conditions. In the following we consider as model for log-prices the following stochastic volatility models, which are Itô semimartingales of the form

$$
Y_{t}=\int_{0}^{t} \sigma_{s} d W_{s}, \quad \check{Y}_{t}=\int_{0}^{t} \sigma_{s} d W_{s}+\int_{0}^{t} d_{s} d s=\int_{0}^{t} \sigma_{s} d W_{s}+D_{t}, \quad 0 \leqslant t \leqslant 1,
$$

where $W$ denotes a standard Brownian motion, $\sigma$ the volatility process and $d$ the drift coefficient. All processes are $\left(\mathcal{F}_{t}\right)$-adapted. With $\breve{Y}$ we want to emphasize that this process has a possibly non vanishing drift term. Without loss of generality we consider as time interval the unit interval [0,1] instead of an interval $[0, T]$ for some $T>0$.

In Palmes and Woerner [9] we considered the following conditions which are a generalization of the conditions in Lee and Mykland [7].

Assumptions 2.1. Let the volatility $\sigma$ be pathwise Hölder continuous, strictly positive and let the drift $d$ be pathwise bounded. This means that there are two functions

$$
\alpha: \Omega \rightarrow(0,1] \text { and } K: \Omega \rightarrow(0, \infty)
$$

such that

$$
\left|\sigma_{t}(\omega)-\sigma_{s}(\omega)\right| \leqslant K(\omega)|t-s|^{\alpha(\omega)}, \quad 0 \leqslant s, t \leqslant 1, \quad \omega \in \Omega
$$

and

$$
\left|\sigma_{t}(\omega)\right| \vee\left|d_{t}(\omega)\right| \leqslant K(\omega), \quad 0 \leqslant t \leqslant 1, \quad \omega \in \Omega
$$

Furthermore, we claim $t \mapsto d_{t}(\omega)$ to be Lebesgue measurable for all $\omega \in \Omega$.

In the following we draw statistical inference with the sampling scheme of high frequency data or the infill asymptotics, i.e. we consider observations at the time-points $0, \frac{1}{N}, \ldots, 1$. However, for our analysis we need a two scale grid which we define as follows. We set $N=n^{2}$ for $n \in \mathbb{N}$ and obtain as sampling times $\frac{l}{n^{2}}, l=0, \ldots, n^{2}-1$ with

$$
\frac{l}{n^{2}}=\frac{k n+j}{n^{2}}=t_{k, j}, \quad 0 \leqslant k, j<n
$$

Hence, the grid on the unit interval separates in two scales. The coarse one, which is indexed by $k$, and the finer one, which is indexed by $j$.

Regarding the increments of the finer scale we define

$$
\Delta W_{k, j}=W_{t_{k, j}+\frac{1}{n^{2}}}-W_{t_{k, j}}, \quad \Delta Y_{k, j}=Y_{t_{k, j}+\frac{1}{n^{2}}}-Y_{t_{k, j}}, \quad \Delta \check{Y}_{k, j}=\check{Y}_{t_{k, j}+\frac{1}{n^{2}}}-\check{Y}_{t_{k, j}}
$$

Setting $Z_{k, j}=n \Delta W_{k, j},\left(Z_{k, j}\right)_{0 \leqslant k, j<n}$ is a family of i.i.d. $\mathrm{N}(0,1)$ (standard normal) distributed random variables, since $W$ is a Brownian motion. Finally, we define some abbreviations concerning the volatility:

$$
\sigma_{k, j}=\sigma_{t_{k, j}}, \quad \sigma_{k}=\sigma_{k, 0} .
$$

The idea of the Gumbel test is that the increments of the log-price process normed with an estimate of the spot volatility behave approximately as i.i.d. standard normal random variables and hence classical extreme value theory may be used. 
Theorem 2.2. [Palmes and Woerner [9]] Set

$$
a_{N}=\sqrt{2 \log N}, \quad b_{N}=a_{N}-\frac{\log (\log N)+\log (4 \pi)}{2 \sqrt{2 \log N}}, \quad N \in \mathbb{N}
$$

and define a statistics $T_{n}$ by

$$
T_{n}=n \max _{0 \leqslant k, j<n}\left(\frac{\Delta \check{Y}_{k, j}}{\check{\sigma}_{k}}\right), \quad n \in \mathbb{N}
$$

with

$$
\breve{\sigma}_{k}^{2}=\frac{\pi n^{2}}{2(n-1)} \sum_{j=0}^{n-2}\left|\Delta \check{Y}_{k, j}\right|\left|\Delta \breve{Y}_{k, j+1}\right|
$$

Then, under the Assumptions 2.1 we obtain for the model with no additive jump component

$$
a_{n^{2}}\left(T_{n}-b_{n^{2}}\right) \stackrel{d}{\rightarrow} \mathcal{G}, \quad n \rightarrow \infty
$$

where $\mathcal{G}$ denotes the Gumbel distribution with the cumulative distribution function $x \mapsto e^{-e^{-x}}, x \in \mathbb{R}$. If the model has an additive jump component $J$ of possibly infinite jump activity and if we replace $\breve{Y}$ in (5) by $\check{Y}+J$, we obtain for $\gamma<\frac{1}{2}$

$$
n^{-\gamma} a_{n^{2}}\left(T_{n}-b_{n^{2}}\right) \rightarrow \infty \quad P \text {-stoch. on } \Lambda,
$$

with

$$
\Lambda=\left\{\omega \in \Omega: \exists t_{0} \in(0,1]: J_{t_{0}}(\omega)-J_{t_{0}-}(\omega)>0\right\} .
$$

Moreover, in the case of a finite jump activity the divergence rate of (7) can be improved from $\sqrt{n}$ to $n$.

Remark 2.3. Theorem 2.2 applies the bipower variation estimator (6). However, in order to keep the technicalities in this paper as small as possible, we will make use of the quadratic variation estimator in (11). Note that there is no need to distinguish between these estimators if we are not concerned with external jumps, cf. Barndorff-Nielsen and Shephard [3]. Merely the divergence rate of $n$ in the case of external jumps with finite activity holds only for the bipower estimator (6). Compare also the results in Palmes and Woerner [9][Section 5] for further details.

The Assumptions 2.1 allow for a fairly general continuous volatility process, namely possible short- and long-range dependence, but jumps in the volatility process are excluded. In the following we will show that the Gumbel test still works under the following condition, i.e. finitely many bounded jumps. Since we are mainly interested in the influence of the jumps in the volatility process we consider the simplifications that the drift $d$ vanishes, $\alpha$ is constant and $W$ and $\sigma$ are independent.

Assumptions 2.4. Let the volatility $\sigma>0$ and the Brownian motion $W$ be independent. Set further $d=0$ and assume

$$
V(\omega)=\inf _{0 \leqslant t \leqslant 1} \sigma_{t}(\omega)>0, \quad \omega \in \Omega .
$$

Furthermore, fix $0 \leqslant \epsilon<1$ and $0<\alpha \leqslant 1$ and let $t \mapsto \sigma_{t}(\omega)$ be càglàd with, at most, finitely many jumps of size not larger than $\epsilon(\sqrt{2}-1) V(\omega)$ and $\alpha$-Hölder continuous between the jumps for every path $\omega \in \Omega$. This means that there is a function $N: \Omega \rightarrow \mathbb{N}_{0}$ and a sequence $\left(S_{l}\right)_{l \geqslant 0}$ of stopping times with $S_{0}=0$,

$$
\begin{cases}S_{l}(\omega)<S_{l+1}(\omega), & 1 \leqslant l \leqslant N(\omega), \\ S_{l}(\omega)=\infty, & l>N(\omega)\end{cases}
$$

such that

$$
\left|\sigma_{s}(\omega)-\sigma_{t}(\omega)\right| \leqslant K(\omega)|t-s|^{\alpha}
$$

holds for all

$$
(s, t) \in \bigcup_{l=0}^{N(\omega)}\left(S_{l}(\omega), S_{l+1}(\omega) \wedge 1\right]^{2}, \quad \omega \in \Omega .
$$


Here, $N$ denotes the number of jumps in the respective path and $\left(S_{l}\right)_{1 \leqslant l \leqslant N}$ are the jump positions. Furthermore, we claim

$$
0<\left|\Delta \sigma_{S_{l}}(\omega)\right|=\left|\sigma_{S_{l}}(\omega)-\sigma_{S_{l}-}(\omega)\right| \leqslant \epsilon(\sqrt{2}-1) V(\omega), \quad 1 \leqslant l \leqslant N, \quad \omega \in \Omega
$$

and assume as usual

$$
\left|\sigma_{t}(\omega)\right|<K(\omega), \quad 0 \leqslant t \leqslant 1, \quad \omega \in \Omega
$$

In general, the Assumptions 2.4 say that sufficiently small jumps with finite activity in the volatility process are allowed. It turns out that the bound of the jump size $(\sqrt{2}-1) V$ is sharp in the sense of Corollary 3.5 in the following section.

For completeness, a formal proof is provided in the Appendix that such a sequence of stopping times $\left(S_{l}\right)_{l \geqslant 0}$ as stated in the Assumptions 2.4 exists, $N$ is measurable and that $K$ can be chosen as a measurable function. This is also important for the proof of Theorem 3.2.

\section{The Gumbel test with jumps in the volatility process}

In this section we will show that under the Assumptions 2.4, i.e. finitely many sufficient small jumps, the Gumbel test is still applicable. Furthermore, we will construct a counter example demonstrating that the bound on the size of the jumps is sharp. We start with a Lemma that builds a bridge between volatility processes with and without jumps. Now we assume w.l.o.g. $P\left(S_{1}=0\right)=0$ and set $K_{l, n} \stackrel{\text { def }}{=}$ $\left(\left\lceil n S_{l}\right\rceil-1\right) \mathbb{1}_{\{l \leqslant N\}}+n \mathbb{1}_{\{l>N\}}, \quad l, n \geqslant 1$.

Lemma 3.1. Under the Assumptions 2.4, we obtain for every $\gamma<\alpha$

$$
n^{\gamma} \max _{k^{\prime}, j}\left|n \Delta Y_{k^{\prime}, j}-\sigma_{k^{\prime}} Z_{k^{\prime}, j}\right| \rightarrow 0, \quad n \rightarrow \infty, \quad P-a . s .
$$

where $k^{\prime}$ in the above maximum runs over all positions of the coarser scaled grid in between the volatility does not possess a jump, i.e.

$$
k^{\prime} \in\{0,1, \ldots, n-1\}-\bigcup_{l=1}^{N}\left\{K_{l, n}\right\}
$$

and $j \in\{0, \ldots, n-1\}$. Hence, the above index set is a random subset of $\{0,1, \ldots, n-1\}^{2}$. If in addition $\gamma<\alpha \wedge \frac{1}{2}$, we obtain

$$
n^{\gamma} \max _{k^{\prime}}\left|\hat{\sigma}_{k^{\prime}}^{2}-\sigma_{k^{\prime}}^{2}\right| \rightarrow 0, \quad n \rightarrow \infty, \quad P \text {-a.s. }
$$

and

$$
n^{\gamma} \max _{k^{\prime}, j}\left|\frac{n \Delta Y_{k^{\prime}, j}}{\hat{\sigma}_{k^{\prime}}}-Z_{k^{\prime}, j}\right| \rightarrow 0, \quad n \rightarrow \infty, \quad \text { P-a.s. }
$$

with the spot volatility estimator

$$
\widehat{\sigma}_{k}^{2}=n \sum_{j=0}^{n-1}\left|\Delta Y_{k, j}\right|^{2}, \quad 0 \leqslant k<n .
$$

Proof. Set

$$
\tau_{t}=\left(1+\sum_{l=1}^{\infty}\left|\Delta \sigma_{S_{l}}\right|\right)-\sum_{l=1}^{\infty} \Delta \sigma_{S_{l}} \mathbb{1}_{\left(S_{l}, 1\right]}(t), \quad 0 \leqslant t \leqslant 1 .
$$

The first term in (12) guarantees that $\tau$ is positive and the second term subtracts all jumps within a path. Thus, $\varphi_{t} \stackrel{\text { def }}{=} \sigma_{t}+\tau_{t}$ is a positive $\alpha$-Hölder continuous process that is independent of $W$. Set further

$$
X_{t}=\int_{0}^{t} \varphi_{s} d W_{s}, \quad C_{t}=\int_{0}^{t} \tau_{s} d W_{s}
$$


Since the volatility $\sigma$ and the Brownian motion $W$ are independent according to the Assumptions 2.4, we can assume w.l.o.g.

$$
\Delta Y_{k, j}=\left(\int_{t_{k, j}}^{t_{k, j}+\frac{1}{n^{2}}} \sigma_{s}^{2} d s\right)^{\frac{1}{2}} Z_{k, j}, \quad 0 \leqslant j, k<n,
$$

cf. Palmes [8][p. 26] for a formal justification.

We start with the proof of (8) : Using

$$
\Delta Y_{k^{\prime}, j}=\Delta X_{k^{\prime}, j}-\Delta C_{k^{\prime}, j}=\Delta X_{k^{\prime}, j}-\tau_{k^{\prime}} \Delta W_{k^{\prime}, j}, \quad \sigma_{k^{\prime}}=\varphi_{k^{\prime}}-\tau_{k^{\prime}},
$$

we have

$$
\left|n \Delta Y_{k^{\prime}, j}-\sigma_{k^{\prime}} Z_{k^{\prime}, j}\right|=\left|n \Delta X_{k^{\prime}, j}-\varphi_{k^{\prime}} Z_{k^{\prime}, j}\right|
$$

which implies

$$
n^{\gamma} \max _{k^{\prime}, j}\left|n \Delta Y_{k^{\prime}, j}-\sigma_{k^{\prime}} Z_{k^{\prime}, j}\right| \leqslant n^{\gamma} \max _{0 \leqslant k, j<n}\left|n \Delta X_{k, j}-\varphi_{k} Z_{k, j}\right| \rightarrow 0, \quad P \text {-a.s. }
$$

Here, the latter convergence holds, since we can apply Palmes and Woerner [9][Proposition 3.3, eq. (12)] to the process $X$.

Next we turn to the proof of (9) : Set

$$
\hat{\varphi}_{k}^{2}=n \sum_{j=0}^{n-1}\left|\Delta X_{k, j}\right|^{2}, \quad \hat{\tau}_{k}^{2}=n \sum_{j=0}^{n-1}\left|\Delta C_{k, j}\right|^{2}, \quad 0 \leqslant k<n
$$

and consider

$$
\begin{aligned}
\hat{\sigma}_{k^{\prime}}^{2} & =\hat{\varphi}_{k^{\prime}}^{2}+\widehat{\tau}_{k^{\prime}}^{2}-2 n \sum_{j=0}^{n-1} \Delta X_{k^{\prime}, j} \Delta C_{k^{\prime}, j} \\
& =\hat{\varphi}_{k^{\prime}}^{2}+\tau_{k^{\prime}}^{2} \frac{1}{n} \sum_{j=0}^{n-1} Z_{k^{\prime}, j}^{2}-2 n \tau_{k^{\prime}} \frac{1}{n} \sum_{j=0}^{n-1} \Delta X_{k^{\prime}, j} Z_{k^{\prime}, j} .
\end{aligned}
$$

Next, decompose the last term in

$$
-2 \tau_{k^{\prime}} \varphi_{k^{\prime}} \frac{1}{n} \sum_{j=0}^{n-1} Z_{k^{\prime}, j}^{2}-2 \tau_{k^{\prime}} R_{k^{\prime}}
$$

with

$$
R_{k^{\prime}} \stackrel{\text { def }}{=} \frac{1}{n} \sum_{j=0}^{n-1} Z_{k^{\prime}, j}\left(n \Delta X_{k^{\prime}, j}-\varphi_{k^{\prime}} Z_{k^{\prime}, j}\right)
$$

Then, we have

$$
\hat{\sigma}_{k^{\prime}}^{2}-\sigma_{k^{\prime}}^{2}=\hat{\sigma}_{k^{\prime}}^{2}-\left(\varphi_{k^{\prime}}-\tau_{k^{\prime}}\right)^{2}=\left(\hat{\varphi}_{k^{\prime}}^{2}-\varphi_{k^{\prime}}^{2}\right)+\left(\tau_{k^{\prime}}^{2}-2 \tau_{k^{\prime}} \varphi_{k^{\prime}}\right)\left(\frac{1}{n} \sum_{j=0}^{n-1} Z_{k^{\prime}, j}^{2}-1\right)-2 \tau_{k^{\prime}} R_{k^{\prime}} .
$$

Again, an application of Palmes and Woerner [9][Propositon 3.3, eq. (13)] to $X$ and $W$ yields

$$
n^{\gamma} \max _{k}\left|\hat{\varphi}_{k}^{2}-\varphi_{k}^{2}\right| \rightarrow 0, \quad \max _{k}\left|\frac{1}{n} \sum_{j=0}^{n-1} Z_{k, j}^{2}-1\right| \rightarrow 0, \quad n \rightarrow \infty, \quad P \text {-a.s. } \quad \text { (resp.) }
$$

Note that [9][eq. (13)] is proven for the bipower variation estimator. However, a simple check of the respective proofs in [9] yields that [9][eq. (13)] still remains valid in the case of the quadratic variation estimator, cf. Palmes [8][Theorem 1.4.2] for more details. 
Note that every path of $\tau$ and $\varphi$ is bounded. Thus, it remains to estimate the residual term $R_{k^{\prime}}$ :

$$
n^{\gamma} \max _{k}\left|R_{k}\right| \leqslant \max _{k} \frac{1}{n}\left|\sum_{j=0}^{n-1} Z_{k, j}\right| n^{\gamma} \max _{k, j}\left|n \Delta X_{k, j}-\varphi_{k} Z_{k, j}\right| .
$$

The second factor converges $P$-a.s. to zero due to Palmes and Woerner [9][Proposition 3.3 eq. (12)]. Note for the first factor

$$
\left(\frac{1}{\sqrt{n}} \sum_{j=0}^{n-1} Z_{k, j}\right)_{k=0, \ldots, n-1} \sim\left(Z_{0, j}\right)_{j=0, \ldots, n-1},
$$

so we have for every $N \in \mathbb{N}$ together with the Markov inequality

$$
P\left(\max _{k} \frac{1}{n}\left|\sum_{j=0}^{n-1} Z_{k, j}\right| \geqslant \epsilon\right)=P\left(\max _{j}\left|Z_{0, j}\right| \geqslant \sqrt{n} \epsilon\right) \leqslant \frac{E\left(\max _{j}\left|Z_{0, j}\right|^{N}\right)}{n^{\frac{N}{2}} \epsilon^{N}}=O\left(n^{-\frac{N-1}{2}}\right) \epsilon^{-N},
$$

where the last equality follows immediately from Palmes and Woerner [8][Proposition A.3]. Hence, a standard Borel-Cantelli argument yields that also the first factor converges $P$-a.s. to zero and, therefore, (9) is proven.

Having proven (8) and (9), the proof of (10) is the same as the proof of the analogue statement in Palmes and Woerner [9][Proposition 4.1] in the case of no jumps in the volatility process. It is therefore omitted.

Theorem 3.2. Theorem 2.2 holds under the Assumptions 2.4 with the spot volatility estimator given by (11).

Proof. Note that the interesting part of the proof is the convergence to the Gumbel distribution, since the other part of the convergence to infinity is not influenced by the conditions on the volatility process.

Hence we prove

$$
a_{n^{2}}\left(T_{n}-b_{n^{2}}\right) \stackrel{\mathrm{d}}{\rightarrow} \mathcal{G}, \quad n \rightarrow \infty .
$$

Lemma 3.1, (10) and

$$
\begin{aligned}
& \left|\max _{k, j} \frac{n \Delta Y_{k, j}}{\hat{\sigma}_{k}}-\max _{k, j} Z_{k, j}\right| \\
\leqslant & \max _{k^{\prime}, j}\left|\frac{n \Delta Y_{k^{\prime}, j}}{\hat{\sigma}_{k^{\prime}}}-Z_{k^{\prime}, j}\right|+\left(\max _{k, j} \frac{n \Delta Y_{k, j}}{\hat{\sigma}_{k}}-\max _{k^{\prime}, j} \frac{n \Delta Y_{k^{\prime}, j}}{\hat{\sigma}_{k^{\prime}}}\right)+\left(\max _{k, j} Z_{k, j}-\max _{k^{\prime}, j} Z_{k^{\prime}, j}\right), \quad n \in \mathbb{N}
\end{aligned}
$$

implies that it suffices to establish the following two convergence results:

$$
P\left(\max _{k, j} \frac{n \Delta Y_{k, j}}{\hat{\sigma}_{k}}-\max _{k^{\prime}, j} \frac{n \Delta Y_{k^{\prime}, j}}{\hat{\sigma}_{k^{\prime}}}>0\right) \rightarrow 0, \quad n \rightarrow \infty
$$

and

$$
P\left(\max _{k, j} Z_{k, j}-\max _{k^{\prime}, j} Z_{k^{\prime}, j}>0\right) \rightarrow 0, \quad n \rightarrow \infty .
$$

The following proof is divided into three steps. The first two steps prove (15). The first step simplifies the claim to a more elementary result which involves only the maximum of i.i.d. $\mathrm{N}(0,1)$ random variables. This simplified result is proven in the second step. Finally, in the third step, the first two steps are used in order to prove (16).

STEP 1. Simplification of the claim. Set

$$
\eta_{k}=\frac{\hat{\sigma}_{k}-\sigma_{k}}{\sigma_{k}}, \quad \zeta_{k, j}=n \Delta Y_{k, j}-\sigma_{k} Z_{k, j}, \quad 0 \leqslant k, j<n
$$


and define for any fixed $0<\gamma<\alpha \wedge \frac{1}{2}$

$$
\begin{aligned}
& A_{n}=\bigcap_{l=0}^{N-1}\left\{S_{l+1}-S_{l}>\frac{1}{n}\right\} \cap\left\{V>\left(2 K n^{-\frac{\alpha}{2}}\right) \vee n^{-\frac{\gamma}{2}}\right\}, \\
& B_{n}=\left\{\max _{k}\left|\frac{1}{n} \sum_{j=0}^{n-1} Z_{k, j}^{2}-1\right| \leqslant \frac{1}{n^{\gamma}}, \max _{k^{\prime}}\left|\eta_{k^{\prime}}\right| \leqslant \frac{1}{\eta^{\gamma}}, \max _{k^{\prime}, j}\left|\zeta_{k^{\prime}, j}\right| \leqslant \frac{1}{n^{\gamma}}, \max _{k, j} Z_{k, j}>0\right\},
\end{aligned}
$$

with the notation $\left\{M_{1}, M_{2}\right\} \stackrel{\text { def }}{=} M_{1} \cap M_{2}$ for any sets $M_{1}, M_{2}$. Furthermore, we have with

$$
\widetilde{k} \in \bigcup_{l=1}^{N}\left\{K_{l, n}\right\}
$$

the inequality

$$
\begin{aligned}
& P\left(\max _{k, j} \frac{n \Delta Y_{k, j}}{\hat{\sigma}_{k}}-\max _{k^{\prime}, j} \frac{n \Delta Y_{k^{\prime}, j}}{\hat{\sigma}_{k^{\prime}}}>0\right) \\
\leqslant & P\left(\max _{\widetilde{k}, j} \frac{n \Delta Y_{\widetilde{k}, j}}{\hat{\sigma}_{\widetilde{k}}}>\max _{k^{\prime}, j} \frac{n \Delta Y_{k^{\prime}, j}}{\widehat{\sigma}_{k^{\prime}}}, A_{n}, B_{n}\right)+P\left(A_{n}^{c}\right)+P\left(B_{n}^{c}\right) .
\end{aligned}
$$

Lemma 10, (9), (10) and (14) yield $P\left(B_{n}^{c}\right) \rightarrow 0, n \rightarrow \infty$. Note also

$$
\limsup P\left(A_{n}^{c}\right) \leqslant P\left(\limsup A_{n}^{c}\right)=P\left(\left(\liminf A_{n}\right)^{c}\right)=P(\varnothing)=0,
$$

i.e. $P\left(A_{n}^{c}\right) \rightarrow 0, n \rightarrow \infty$. Set $G_{l, n} \stackrel{\text { def }}{=}\left\{\left(g_{1}, \ldots, g_{l}\right) \in\{0,1, \ldots, n-1\}^{l}: g_{1}<g_{2}<\ldots<g_{l}\right\}, \quad 1 \leqslant l \leqslant n$. Since we aim to verify (15), the above yields that it is sufficient to prove that

$$
\sum_{l=1}^{n} \sum_{g \in G_{l, n}} P\left(\max _{\widetilde{k}, j} \frac{n \Delta Y_{\widetilde{k}, j}}{\hat{\sigma}_{\widetilde{k}}}-\max _{k^{\prime}, j} \frac{\Delta Y_{k^{\prime}, j}}{\widehat{\sigma}_{k^{\prime}}}>0,\left(K_{1, n}, \ldots, K_{l, n}\right)=g, N=l, A_{n}, B_{n}\right)
$$

tends to zero, if $n$ tends to infinity. Define

$$
\begin{aligned}
\lambda_{k} & =\inf _{\left\{\frac{k}{n} \leqslant s \leqslant \frac{k+1}{n}\right\}} \sigma_{s}, \quad 0 \leqslant k<n, \\
\delta & =\sup _{l \geqslant 1}\left|\Delta \sigma_{S_{l}}\right| \mathbb{1}_{\left\{S_{l} \leqslant 1\right\}} \leqslant \epsilon(\sqrt{2}-1) V .
\end{aligned}
$$

Then we have for $\omega \in A_{n}$ and every $0 \leqslant k<n$, i.e. in particular for $\widetilde{k}$

$$
\lambda_{k}(\omega) \leqslant \sigma_{s}(\omega) \leqslant \lambda_{k}(\omega)+2 K(\omega) n^{-\alpha}+\delta(\omega) \stackrel{\text { def }}{=} \lambda_{k}(\omega)+\delta_{n}(\omega), \quad \frac{k}{n} \leqslant s \leqslant \frac{k+1}{n} .
$$

This yields the estimates

$$
\lambda_{k}^{2} \frac{1}{n} \sum_{j=0}^{n-1} Z_{k, j}^{2} \leqslant \hat{\sigma}_{k}^{2} \leqslant\left(\lambda_{k}+\delta_{n}\right)^{2} \frac{1}{n} \sum_{j=0}^{n-1} Z_{k, j}^{2}, \quad \omega \in A_{n},
$$

since

$$
\hat{\sigma}_{k}^{2}=n \sum_{j=0}^{n-1}\left(\Delta Y_{k, j}\right)^{2}=n \sum_{j=0}^{n-1} \int_{t_{k, j}}^{t_{k, j}+\frac{1}{n^{2}}} \sigma_{s}^{2} d s Z_{k, j}^{2}
$$

cf. (13). Using this we obtain with $C_{n} \stackrel{\text { def }}{=} A_{n} \cap B_{n}, n \in \mathbb{N}$ the inequalities

$$
P\left(\max _{\widetilde{k}, j} \frac{n \Delta Y_{\widetilde{k}, j}}{\hat{\sigma}_{\widetilde{k}}}>\max _{k^{\prime}, j} \frac{n \Delta Y_{k^{\prime}, j}}{\widehat{\sigma}_{k^{\prime}}},\left(K_{1, n}, \ldots, K_{l, n}\right)=g, N=l, C_{n}\right)
$$




$$
\begin{aligned}
& \leqslant P\left(\max _{\widetilde{k}, j} \frac{n\left(\frac{1}{n^{2}}\left(\lambda_{\widetilde{k}}+\delta_{n}\right)^{2}\right)^{\frac{1}{2}} Z_{\widetilde{k}, j}}{\left(\lambda_{\widetilde{k}}^{2}\left(1-\frac{1}{n^{\gamma}}\right)\right)^{\frac{1}{2}}}>\max _{k^{\prime}, j} \frac{\sigma_{k^{\prime}} Z_{k^{\prime}, j}+\zeta_{k^{\prime}, j}}{\sigma_{k^{\prime}}\left(1+\eta_{k^{\prime}}\right)},\left(K_{1, n}, \ldots, K_{l, n}\right)=g, N=l, C_{n}\right) \\
& \leqslant P\left(\max _{\widetilde{k}, j} \frac{\lambda_{\widetilde{k}}+\delta_{n}}{\lambda_{\widetilde{k}}} \frac{Z_{\widetilde{k}, j}}{\left(1-\frac{1}{n^{\gamma}}\right)^{\frac{1}{2}}}>\max _{k^{\prime}, j} \frac{Z_{k^{\prime}, j}}{1+\frac{1}{n^{\gamma}}}-\frac{\frac{1}{n^{\gamma}}}{V\left(1-\frac{1}{n^{\gamma}}\right)},\left(K_{1, n}, \ldots, K_{l, n}\right)=g, N=l, C_{n}\right) .
\end{aligned}
$$

We have on $A_{n}$ for $n$ large enough

$$
\frac{\lambda_{\tilde{k}}+\delta_{n}}{\lambda_{\widetilde{k}}} \leqslant 1+\frac{n^{-\frac{\alpha}{2}} V+\epsilon(\sqrt{2}-1) V}{V}=\sqrt{2}+n^{-\frac{\alpha}{2}}-(\sqrt{2}-1)(1-\epsilon) \leqslant \sqrt{2-\kappa}
$$

for some constant $\kappa>0$. Hence, if $g \in G_{l, n}, l \leqslant n$, (18) is not larger than

$$
P\left(\frac{\sqrt{2-\kappa}}{\left(1-\frac{1}{n^{\gamma}}\right)^{\frac{1}{2}}} \max _{\widetilde{k}, j} Z_{\widetilde{k}, j}>\frac{1}{1+\frac{1}{n^{\gamma}}} \max _{k^{\prime}, j} Z_{k^{\prime}, j}-\frac{2}{n^{\frac{\gamma}{2}}}\right) P\left(\left(K_{1, n}, \ldots, K_{l, n}\right)=g, N=l\right)
$$

where the maxima run over

$$
\tilde{k} \in \bigcup_{j=1}^{l}\left\{g_{j}\right\} \quad \text { and } \quad k^{\prime} \in\{0,1, \ldots, n-1\}-\bigcup_{j=1}^{l}\left\{g_{j}\right\},
$$

respectively. Here, we used the independence of $Z$ and $\sigma$. Define

$$
D_{l, n}^{g}=\left\{\frac{\sqrt{2-\kappa}}{\left(1-\frac{1}{n^{\gamma}}\right)^{\frac{1}{2}}} \max _{\widetilde{k}, j} Z_{\widetilde{k}, j}>\frac{1}{1+\frac{1}{n^{\gamma}}} \max _{k^{\prime}, j} Z_{k^{\prime}, j}-\frac{2}{n^{\frac{\gamma}{2}}}\right\}, \quad l \leqslant n, g \in G_{l, n} .
$$

It suffices to prove $P\left(D_{l, n}^{\pi}\right) \rightarrow 0, n \rightarrow \infty$ with $\pi=(0,1, \ldots, l-1) \in G_{l, n}$ for every fixed $l \leqslant n$, since we have

$$
\begin{aligned}
& \sum_{l=1}^{n} \sum_{g \in G_{l, n}} P\left(D_{l, n}^{g}\right) P\left(\left(K_{1, n}, \ldots, K_{l, n}\right)=g, N=l\right) \\
= & \sum_{l=1}^{n} P\left(D_{l, n}^{\pi}\right) \sum_{g \in G_{l, n}} P\left(\left(K_{1, n}, \ldots, K_{l, n}\right)=g, N=l\right) \\
\leqslant & \sum_{l=1}^{n} P\left(D_{l, n}^{\pi}\right) P(N=l)
\end{aligned}
$$

and

$$
P\left(D_{l, n}^{\pi}\right) \leqslant 1, \quad \sum_{l=1}^{\infty} P(N=l) \leqslant 1<\infty .
$$

Hence, a dominated convergence argument, (17) and the results proven so far yield the desired convergence (15).

STEP 2. Convergence of $\left(D_{l, n}^{\pi}\right)_{n}$. We know that

$$
\alpha_{l, n} \stackrel{\text { def }}{=} \max _{\widetilde{k}, j} Z_{\widetilde{k}, j}-b_{l n} \rightarrow 0, \quad \beta_{l, n} \stackrel{\text { def }}{=} \max _{k^{\prime}, j} Z_{k^{\prime}, j}-b_{(n-l) n} \rightarrow 0, \quad n \rightarrow \infty \quad P \text {-stoch. }
$$

Due to

$$
\begin{aligned}
& P\left(D_{l, n}^{\pi}\right) \\
= & P\left(\frac{\sqrt{2-\kappa}}{\left(1-\frac{1}{n^{\gamma}}\right)^{\frac{1}{2}}}\left(\alpha_{l, n}+b_{l n}\right)>\frac{1}{1+\frac{1}{n^{\gamma}}}\left(\beta_{l, n}+b_{(n-l) n}\right)-\frac{2}{n^{\frac{\gamma}{2}}}\right)
\end{aligned}
$$




$$
=P\left(\frac{\sqrt{2-\kappa}}{\left(1-\frac{1}{n^{\gamma}}\right)^{\frac{1}{2}}} \alpha_{l, n}-\frac{1}{1+\frac{1}{n^{\gamma}}} \beta_{l, n}>\frac{1}{1+\frac{1}{n^{\gamma}}} b_{(n-l) n}-\frac{\sqrt{2-\kappa}}{\left(1-\frac{1}{n^{\gamma}}\right)^{\frac{1}{2}}} b_{l n}-\frac{2}{n^{\frac{\gamma}{2}}}\right)
$$

and the stochastic convergence of $\left(\alpha_{l, n}\right)_{n}$ and $\left(\beta_{l, n}\right)_{n}$ to zero, it suffices to show

$$
\frac{1}{1+\frac{1}{n^{\gamma}}} b_{(n-l) n}-\frac{\sqrt{2-\kappa}}{\left(1-\frac{1}{n^{\gamma}}\right)^{\frac{1}{2}}} b_{l n} \rightarrow \infty, \quad n \rightarrow \infty .
$$

Substituting (4) in (19) yields

$$
\begin{aligned}
& \frac{1}{1+\frac{1}{n^{\gamma}}} \sqrt{2 \log ((n-l) n)}-\sqrt{\frac{2-\kappa}{1-\frac{1}{n^{\gamma}}}} \sqrt{2 \log (\ln )}+o(1), \quad n \rightarrow \infty \\
= & \sqrt{2 \log \left(((n-l) n)^{\frac{1}{\left(1+\frac{1}{n^{\gamma}}\right)^{2}}}\right)}-\sqrt{2 \log \left((\ln )^{\frac{2-\kappa}{1-\frac{1}{n^{\gamma}}}}\right)}+o(1) .
\end{aligned}
$$

Since

$$
\frac{1}{\left(1+\frac{1}{n^{\gamma}}\right)^{2}} \rightarrow 1, \quad \frac{2-\kappa}{1-\frac{1}{n^{\gamma}}} \rightarrow 2-\kappa<2, \quad n \rightarrow \infty
$$

step 2 is completed.

STEP 3. Proof of (16). Write as at the end of step 1

$$
\begin{aligned}
& P\left(\max _{k, j} Z_{k, j}-\max _{k^{\prime}, j} Z_{k^{\prime}, j}>0, A_{n}\right) \\
\leqslant & \sum_{l=1}^{n} \sum_{g \in G_{l, n}} P\left(\max _{\widetilde{k}, j} Z_{\widetilde{k}, j}>\max _{k^{\prime}, j} Z_{k^{\prime}, j},\left(K_{1, n}, \ldots, K_{l, n}\right)=g, N=l\right) \\
= & \sum_{l=1}^{n} \sum_{g \in G_{l, n}} P\left(\max _{\widetilde{k}, j} Z_{\widetilde{k}, j}>\max _{k^{\prime}, j} Z_{k^{\prime}, j}\right) P\left(\left(K_{1, n}, \ldots, K_{l, n}\right)=g, N=l\right) .
\end{aligned}
$$

Again it suffices to establish

$$
P\left(\max _{\widetilde{k}, j} Z_{\widetilde{k}, j}>\max _{k^{\prime}, j} Z_{k^{\prime}, j}\right) \rightarrow 0, \quad n \rightarrow \infty
$$

for every fixed $l \in \mathbb{N}$ and $g \in G_{l, n}$. Note that the proof of (20) is a simpler version of what was performed for the second step, namely set $\kappa=1$.

Next we turn to the question, whether the bound on the size is optimal or if it might be chosen larger. In the following, we demonstrate that the bound $(\sqrt{2}-1) V$ is sharp. Our main result in this context is Corollary 3.5 which is a stochastic generalization of the following Proposition 3.4. We illustrate that the convergence to the Gumbel distribution does not have to hold, if there is an oversized jump in the volatility process at some irrational position. Such an irrational jump position causes some problems since our grid consists of equidistant rational points. We start with the following preparatory lemma:

Lemma 3.3. Fix any $0<c, r<1$ and let $c$ be an irrational number, then there are sequences $\left(n_{l}\right)$ and $\left(k_{l}\right)$ of natural numbers with the properties

$$
n_{l} \uparrow \infty, \quad 0 \leqslant k_{l}<n_{l}
$$

and

$$
\frac{k_{l}}{n_{l}}+\frac{r}{2 n_{l}}<c<\frac{k_{l}}{n_{l}}+\frac{r}{n_{l}}, \quad l \geqslant 1 .
$$


Proof. Consider the function

$$
g: \mathbb{N} \rightarrow[0,1], \quad n \mapsto n c-\lfloor n c\rfloor .
$$

$g(\mathbb{N})$ is a dense subset of $[0,1]$. This is due to the irrationality of $c$ and can be proven by the pigeon-hole principle, c.f. Arnold [2][§24, page 222]. Observe that (21) is the same as claiming

$$
n_{l} c-k_{l} \in\left(\frac{r}{2}, r\right), \quad l \geqslant 1 .
$$

Since $\left(\frac{r}{2}, r\right) \subseteq[0,1]$ is open and $g(\mathbb{N})$ is dense in $[0,1]$, it follows that

$$
g(\mathbb{N}) \cap\left(\frac{r}{2}, r\right) \subseteq[0,1]
$$

consists of infinite many points. So we can choose a sequence $n_{l} \uparrow \infty$ of natural numbers such that

$$
g\left(n_{l}\right) \in\left(\frac{r}{2}, r\right), \quad l \geqslant 1
$$

holds. Finally, we set

$$
0 \leqslant k_{l} \stackrel{\text { def }}{=}\left\lfloor n_{l} c\right\rfloor<n_{l}, \quad l \geqslant 1
$$

and observe that this choice yields the equivalence of (22) and (23) which proves this lemma.

Using this, we can prove the following main result in this context:

Proposition 3.4. Let $h$ and $c$ be two numbers, such that $h>\sqrt{2}$ and $0<c<1$ is an irrational number. Set

$$
\sigma_{t}=h \mathbb{1}_{[0, c]}(t)+\mathbb{1}_{(c, 1]}(t), \quad 0 \leqslant t \leqslant 1
$$

and $d_{t}=0$. Then, there is a sequence $\left(n_{l}\right)_{l}$ of natural numbers, such that $n_{l} \uparrow \infty$ and

$$
P\left(T_{n_{l}}-b_{n_{l}^{2}} \geqslant \epsilon\right) \rightarrow 1, \quad l \rightarrow \infty
$$

for all $\epsilon>0$. This implies in particular

$$
a_{n^{2}}\left(T_{n}-b_{n^{2}}\right)+\mathcal{G}, \quad n \rightarrow \infty .
$$

Proof. We use the fact that the spot volatility estimator $\hat{\sigma}_{k}$ estimates the average value of the spot volatility in the interval $\left[\frac{k}{n}, \frac{k+1}{n}\right]$. Thus, if the spot volatility jumps in this interval, we make obviously an error depending on the jump size and position. Our intention in the following is to make this error as large as possible to get the negative convergence result (24).

The proof is divided into two steps. Similar to the proof of Theorem 3.2, the first step simplifies our claim, so that it remains to prove a more elementary result which involves only the maximum of i.i.d. $\mathrm{N}(0,1)$ random variables. We prove this result in the second step.

STEP 1. Simplification of the claim. We have

$$
Y_{t}=\int_{0}^{t} \sigma_{s} d W_{s}= \begin{cases}h W_{t}, & t \leqslant c \\ W_{t}-W_{c}+h W_{c}, & t>c .\end{cases}
$$

Let

$$
f_{n}(t)=\left\lfloor n^{2} t\right\rfloor-n\lfloor n t\rfloor, \quad 0<t<1
$$

denote the fine scale position of $t$ and choose $0 \leqslant k<n$, such that $c \in\left(\frac{k}{n}, \frac{k+1}{n}\right)$. Then we can write

$$
\hat{\sigma}_{k}^{2}=n \sum_{j=0}^{f_{n}(c)-1}\left(\Delta Y_{k, j}\right)^{2}+\epsilon_{k, n}+n \sum_{j=f_{n}(c)+1}^{n-1}\left(\Delta Y_{k, j}\right)^{2}, \quad 0 \leqslant \epsilon_{k, n} \leqslant \frac{h^{2}}{n} Z_{k, f_{n}(c)}^{2}
$$




$$
=\frac{h^{2}}{n} \sum_{j=0}^{f_{n}(c)-1} Z_{k, j}^{2}+\frac{1}{n} \sum_{j=f_{n}(c)+1}^{n-1} Z_{k, j}^{2}+\epsilon_{k, n}
$$

Next set

$$
r \stackrel{\text { def }}{=} \frac{h-\sqrt{2}}{4\left(h^{2}-1\right)} \in(0,1)
$$

and note that by Lemma 3.3 we have two sequences $\left(n_{l}\right)_{l}$ and $\left(k_{l}\right)_{l}$ of natural numbers, such that

$$
\left\lfloor\frac{r}{2} n_{l}\right\rfloor \leqslant f_{n_{l}}(c) \leqslant\left\lfloor r n_{l}\right\rfloor, \quad c \in\left(\frac{k_{l}}{n_{l}}, \frac{k_{l}+1}{n_{l}}\right), l \in \mathbb{N}
$$

and $n_{l} \uparrow \infty$. This implies together with the weak law of large numbers

$$
\hat{\sigma}_{k_{l}}^{2} \leqslant \frac{h^{2}}{n_{l}} \sum_{j=0}^{\left\lfloor r n_{l}\right\rfloor} Z_{k_{l}, j}^{2}+\frac{1}{n_{l}} \sum_{j=\left\lfloor r n_{l}\right\rfloor+1}^{n_{l}-1} Z_{k_{l}, j}^{2} \rightarrow r h^{2}+(1-r), \quad l \rightarrow \infty \quad(P \text {-stoch. })
$$

which yields

$$
\begin{aligned}
P\left(\hat{\sigma}_{k_{l}}^{2} \geqslant 1+2 r\left(h^{2}-1\right)\right) & \leqslant P\left(\left|\frac{h^{2}}{n_{l}} \sum_{j=0}^{\left\lfloor r n_{l}\right\rfloor} Z_{k_{l}, j}^{2}+\frac{1}{n_{l}} \sum_{j=\left\lfloor r n_{l}\right\rfloor+1}^{n_{l}-1} Z_{k_{l}, j}^{2}-1-r\left(h^{2}-1\right)\right| \geqslant r\left(h^{2}-1\right)\right) \\
& \rightarrow 0, \quad l \rightarrow \infty
\end{aligned}
$$

Next define

$$
\lambda=\frac{h}{1+2 r\left(h^{2}-1\right)}>\sqrt{2}
$$

cf. (25) and

$$
r_{l}=\frac{\left\lfloor\frac{r}{2} n_{l}\right\rfloor}{n_{l}} \geqslant \frac{r}{2}-\frac{1}{n_{l}}, \quad l \geqslant 1 .
$$

Furthermore, note that for arbitrary $\epsilon>0$ we have the inequalities

$$
\begin{aligned}
P\left(T_{n_{l}}-b_{n_{l}^{2}} \geqslant \epsilon\right) & \geqslant P\left(n_{l} \max _{0 \leqslant j<\left\lfloor\frac{r}{2} n_{l}\right\rfloor} \frac{\Delta Y_{k_{l}, j}}{\hat{\sigma}_{k_{l}}}-b_{n_{l}^{2}} \geqslant \epsilon\right) \\
& \geqslant P\left(\lambda \max _{0 \leqslant j<r_{l} n_{l}} Z_{k_{l}, j}-b_{n_{l}^{2}} \geqslant \epsilon, \hat{\sigma}_{k_{l}}^{2} \leqslant 1+2 r\left(h^{2}-1\right)\right) .
\end{aligned}
$$

Thus regarding (26), it suffices to establish

$$
P\left(\lambda \max _{0 \leqslant j<r_{l} n_{l}} Z_{k_{l}, j}-b_{n_{l}^{2}} \geqslant \epsilon\right) \rightarrow 1, \quad l \rightarrow \infty,
$$

which we will show in the second step.

STEP 2. Convergence to infinity of a $\lambda$-scaled partial-maximum. Crucial in what follows is that we have the lower bound $\lambda>\sqrt{2}$, which is due to the choice of $r$. The notations

$$
M_{l} \stackrel{\text { def }}{=} \max _{0 \leqslant j<r_{l} n_{l}} Z_{k_{l}, j}, \quad A_{l} \stackrel{\text { def }}{=} a_{r_{l} n_{l}}\left(M_{l}-b_{r_{l} n_{l}}\right), \quad l \geqslant 1
$$

are used in the following. We know that $A_{l} \stackrel{\mathrm{d}}{\rightarrow} \mathcal{G}, l \rightarrow \infty$, cf. Lemma 1.1.7 in Haan and Ferreira [4], and write

$$
\begin{aligned}
P\left(\lambda M_{l}-b_{n_{l}^{2}} \geqslant \epsilon\right) & =P\left(\lambda\left(A_{l}+a_{r_{l} n_{l}} b_{r_{l} n_{l}}\right)-a_{r_{l} n_{l}} b_{n_{l}^{2}} \geqslant a_{r_{l} n_{l}} \epsilon\right) \\
& =P\left(A_{l} \geqslant \frac{1}{\lambda}\left(a_{r_{l} n_{l}} b_{n_{l}^{2}}+a_{r_{l} n_{l}} \epsilon\right)-a_{r_{l} n_{l}} b_{r_{l} n_{l}}\right) .
\end{aligned}
$$


Obviously it suffices to establish

$$
\frac{1}{\lambda} b_{n_{l}^{2}}-b_{r_{l} n_{l}} \rightarrow-\infty, \quad l \rightarrow \infty .
$$

This follows after a substitution of (4), i.e.

$$
\begin{aligned}
b_{n_{l}^{2}}-\lambda b_{r_{l} n_{l}} & =\sqrt{2 \log n_{l}^{2}}-\lambda \sqrt{2 \log \left(r_{l} n_{l}\right)}+o(1), \quad l \rightarrow \infty \\
& =2 \sqrt{\log n_{l}}-\sqrt{2} \lambda \sqrt{\log r_{l}+\log n_{l}}+o(1) \\
& =2 \sqrt{\log n_{l}}(1-\underbrace{\frac{\lambda}{\sqrt{2}}}_{>1} \underbrace{\sqrt{\frac{\log r_{l}}{\log n_{l}}+1}}_{\rightarrow 1})+o(1) \\
& \rightarrow-\infty, \quad l \rightarrow \infty .
\end{aligned}
$$

Corollary 3.5. Let $\left(\Omega, \mathcal{F},\left(\mathcal{F}_{t}\right)_{0 \leqslant t \leqslant 1}, P\right)$ be a filtered probability space. Furthermore, assume that $W$ is $a\left(\mathcal{F}_{t}\right)$-adapted Brownian motion on this space and that there are two random variables $S, H: \Omega \rightarrow \mathbb{R}$, such that $(S, H)$ is $\mathcal{F}_{0}$ measurable and independent of $W$. Next assume that the distribution of $(S, H)$ has an atom at some point $(c, h)$. To be more precise, let

$$
P((S, H)=(c, h))>0
$$

for some pair $(c, h)$ with

$$
0<c<1, c \notin \mathbb{Q}, \quad h>\sqrt{2} .
$$

Further set

$$
\begin{aligned}
& \sigma_{t}^{(S, H)} \stackrel{\text { def }}{=} H \mathbb{1}_{[0, S]}(t)+\mathbb{1}_{(S, 1]}(t), \\
& Y_{t}^{(S, H)} \stackrel{\text { def }}{=} \int_{0}^{t} \sigma_{s}^{(S, H)} d W_{s}, \quad 0 \leqslant t \leqslant 1
\end{aligned}
$$

and define $\left(T_{n}^{(S, H)}\right)_{n}$ analogue to $\left(T_{n}\right)_{n}$ as a function of $\left(Y_{t}^{(S, H)}\right)_{t}$. Then there is a sequence $\left(n_{l}\right)$ of natural numbers with $n_{l} \uparrow \infty$, such that

$$
P\left(T_{n_{l}}^{(S, H)}-b_{n_{l}^{2}} \geqslant \epsilon\right) \rightarrow 1, \quad l \rightarrow \infty
$$

for all $\epsilon>0$. This implies in particular

$$
a_{n^{2}}\left(T_{n}^{(S, H)}-b_{n^{2}}\right) \rightarrow \mathcal{G}, \quad n \rightarrow \infty .
$$

Remark 3.6. The assumptions of Corollary 3.5 basically state that the volatility jumps at the position $S$ with the jump size $H-1$ if $0<S \leqslant 1$ and $H \neq 1$. Furthermore, there is a positive probability that $\sigma$ jumps at some irrational position with a jump size larger than $\sqrt{2}-1$. Note also that the existence of a filtration as stated in Corollary 3.5 does not cause any problems. This is due to the fact that if $\mathcal{H} \subseteq \mathcal{F}$ is a sub- $\sigma$-algebra, which is independent of $\mathcal{F}_{1}$, then

$$
\left(W_{t}, \mathcal{H}_{t}\right)_{0 \leqslant t \leqslant 1}, \quad \mathcal{H}_{t} \stackrel{\text { def }}{=} \sigma\left(\mathcal{H} \cup \mathcal{F}_{t}\right), \quad 0 \leqslant t \leqslant 1
$$

is also a Brownian motion. We have to consider such sophisticated technicalities since the construction of the Itô integral requires $\sigma$ to be $\left(\mathcal{F}_{t}\right)_{t}$ adapted.

Proof of Corollary 3.5. Using the independence of $(S, H)$ and $W, P((S, H)=(c, h))>0$ and the statement of Proposition 3.4 we can write for any $\epsilon>0$

$$
P\left(T_{n}^{(S, H)}-b_{n^{2}} \geqslant \epsilon\right)=\int_{\mathbb{R}^{2}} P\left(T_{n}^{(S, H)}-b_{n^{2}} \geqslant \epsilon \mid(S, H)=(s, u)\right) d P^{(S, H)}(s, u)
$$




$$
\begin{aligned}
& \geqslant P((S, H)=(c, h)) P\left(T_{n}^{(S, H)}-b_{n^{2}} \geqslant \epsilon \mid(S, H)=(c, h)\right) \\
& =P((S, H)=(c, h)) P\left(T_{n}^{(c, h)}-b_{n^{2}} \geqslant \epsilon\right) .
\end{aligned}
$$

This implies with the same sequence $\left(n_{l}\right)_{l}$ as in Proposition 3.4 the convergence

$$
P\left(T_{n_{l}}^{(S, H)}-b_{n_{l}^{2}} \geqslant \epsilon\right) \rightarrow 1, \quad l \rightarrow \infty .
$$

Furthermore, (27) follows from Slutsky's theorem together with $a_{n^{2}} \uparrow \infty$ as $n \rightarrow \infty$.

\section{Simulation study}

Theorem 3.2 claims that our scaled test statistics (5) still converges to the Gumbel distribution in the presence of sufficiently small jumps, not larger than the bound $V(\sqrt{2}-1)$, cf. Assumptions 2.4. Furthermore, Corollary 3.5 states that this bound is sharp, i.e. it is the lowest possible bound.

In this section, we investigate the convergence respectively divergence properties of the scaled statistics (5) by numerical simulations in the case that the volatility process possesses jumps of the critical size. Our simulations clearly confirm the existence of such a critical jump size. Hence, the simulations coincide with our theoretical investigations of the previous section.
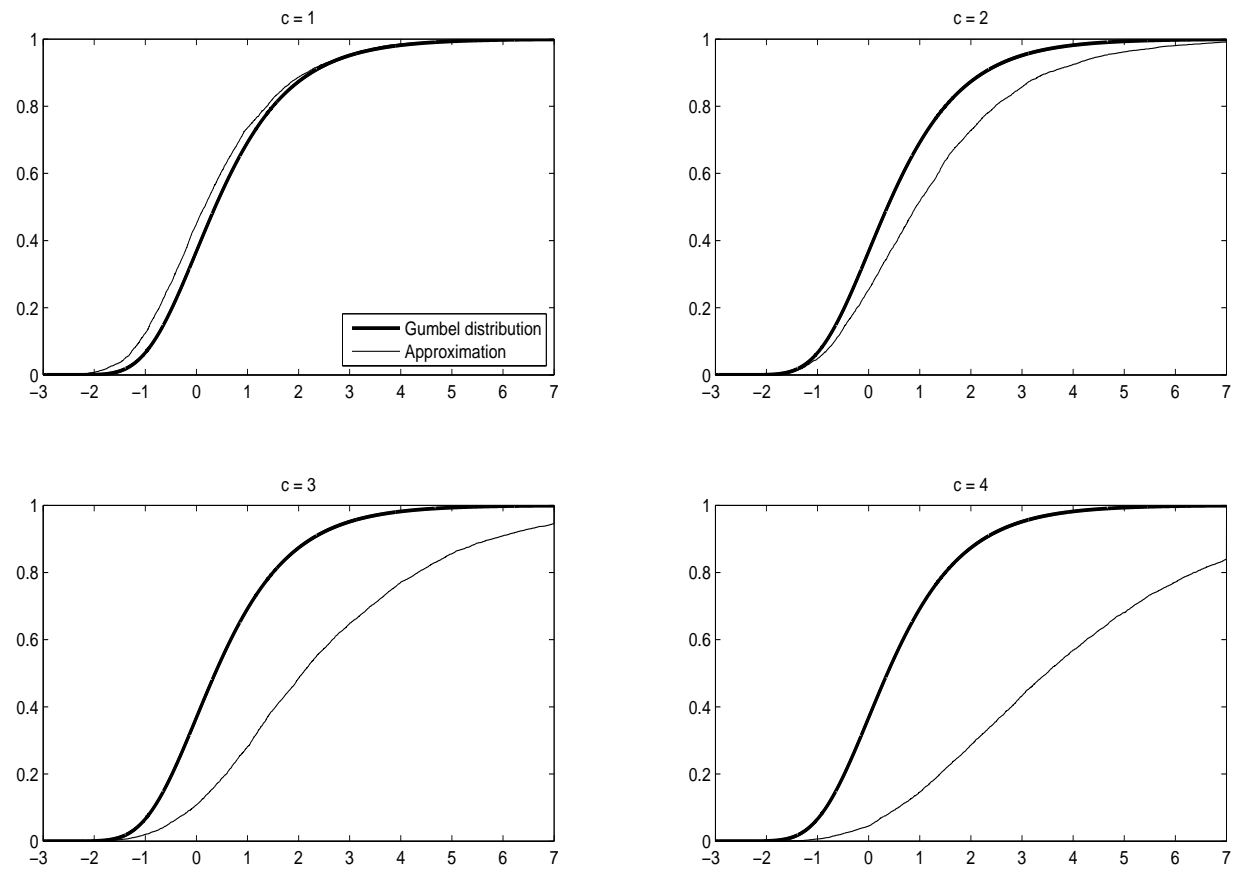

Figure 1: A sharp bound concerning the jump size of the volatility process

In what follows, we construct a suitable volatility process. Fix $n \in \mathbb{N}$ and $0<p<1$ and let $X_{0}, \ldots, X_{n-1}$ be a family of i.i.d. Bernoulli distributed random variables with success probability $p$. Moreover, let $U_{0}, \ldots, U_{n-1}$ be i.i.d. and uniformly distributed on $[0,1]$ and let $B$ and $W$ be two standard Brownian motions, such that $X, U, B, W$ are independent. Set further

$$
\tau_{t}=1+\left|1+B_{t}\right|, \quad 0 \leqslant t \leqslant 1, \quad \lambda_{k}=\inf _{\frac{k}{n} \leqslant s \leqslant \frac{k+1}{n}} \tau_{s}, \quad 0 \leqslant k<n,
$$




$$
\begin{aligned}
\gamma_{t}^{(n)} & =\sum_{k=0}^{\lfloor n t\rfloor-1} \lambda_{k} \mathbb{1}_{\{1\}}\left(X_{k}\right)+\lambda_{\lfloor n t\rfloor} \mathbb{1}_{\{1\} \times(0, n t-\lfloor n t\rfloor)}\left(X_{\lfloor n t\rfloor}, U_{\lfloor n t\rfloor}\right), \quad \gamma_{1}^{(n)}=0, \quad 0 \leqslant t<1, \\
\sigma_{t}^{(c, n)} & =\tau_{t}+c(\sqrt{2}-1) \gamma_{t}^{(n)}, \quad 0 \leqslant t \leqslant 1, \quad c>0
\end{aligned}
$$

and

$$
Y_{t}^{(c, n)}=\int_{0}^{t} \sigma_{s}^{(c, n)} d W_{s}, \quad 0 \leqslant t \leqslant 1 .
$$

$\tau$ is a reflected Brownian motion with starting point $\tau_{0}=2$ and reflection barrier 1. Further, $t \mapsto \gamma_{t}^{(n)}$ is a step function that jumps on every subinterval $I_{k} \stackrel{\text { def }}{=}\left[\frac{k}{n}, \frac{k+1}{n}\right]$ with probability $p$, i.e. iff $X_{k}=1$. In this case, the jump position is uniformly distributed on $I_{k}$ according to $U_{k}$, and the jump size is the infimum of $\tau$ in $I_{k}$. Thus, (28) adds on $\tau$ on every coarse grid position with probability $p$ a jump of the critical size $c V(\sqrt{2}-1)$. Therefore, every path of $\sigma^{(c, n)}$ has a $\operatorname{Bin}(n, p)$ distributed number of jumps.

We used in our simulations the grid-size $\frac{1}{n^{2}}$ with $n=200$ and simulated $Y^{(c, n)}$ with $c=1,2,3,4$ and $p=0.1$, i.e. every volatility path possesses on average $n p=20$ jumps. The scaled test statistics (5) was evaluated 5000 times for each $c$ and the resulting empirical distribution functions are plotted together with the Gumbel distribution in Figure 1. The divergence (to infinity) away from the the Gumbel distribution is clearly observed in the cases $c=2,3,4$. Otherwise, the resulting plot of $c=1$ is close to the plot of the Gumbel distribution. This confirms our theoretical result that there exists such a jump size bound of the volatility process.

\section{Appendix}

For completeness, we provide a proof that a sequence of stopping times $\left(S_{l}\right)_{l \geqslant 0}$ as stated in the Assumptions 2.4 exists, $N$ is measurable and that $K$ can be chosen as a measurable function. Assume for this purpose that the Assumptions 2.4 hold and set for $l \geqslant 1$

$$
S_{l}(\omega)= \begin{cases}\text { Position of the } l \text {-th jump in } \sigma(\omega), & \sigma(\omega) \text { has at least } l \text { jumps }, \\ \infty, & \text { else }\end{cases}
$$

and set $S_{0}=0$. To understand that each $S_{l}$ is a stopping time, an inductive argument is provided. Firstly, define for $r, s, u, v \in \mathbb{Q}$ and $m, n \in \mathbb{N}$ the sets

$$
\begin{aligned}
I_{u, v, n} & =\left\{(r, s) \in \mathbb{Q}^{2}: u<r, s<v \text { and }|r-s|<\frac{1}{n}\right\} \\
A_{r, s, m} & =\left\{\omega \in \Omega:\left|\sigma_{r}(\omega)-\sigma_{s}(\omega)\right|>\frac{1}{m}\right\} .
\end{aligned}
$$

$S_{0}$ is obviously a stopping time. Assume for the induction step that $S_{l}$ is also a stopping time for some $l \in \mathbb{N}$. Observe, furthermore, for $t>0$ and

$$
C_{u, v}=\bigcup_{m \in \mathbb{N}} \bigcap_{n \in \mathbb{N}} \bigcup_{(r, s) \in I_{u, v, n}} A_{r, s, m} \in \mathcal{F}_{v}, \quad 0<u, v<1
$$

the relation

$$
\left\{S_{l+1}<t\right\}=\bigcup_{\substack{0<s<t, s \in \mathbb{Q}}}\left\{S_{l}<s\right\} \cap C_{s, t} \in \mathcal{F}_{t}
$$

which proves that $S_{l+1}$ is a stopping time due to the right continuity of the filtration $\left(\mathcal{F}_{t}\right)$. Next

$$
\{N=n\}=\left\{S_{n}<\infty\right\} \cap\left\{S_{n+1}=\infty\right\} \in \mathcal{F}, \quad n \in \mathbb{N}_{0}
$$

yields that $N$ is measurable. It remains to establish that $K$ can be chosen as a measurable function. To understand this, set

$$
\psi_{t}=\sigma_{t}-\sum_{l=1}^{\infty} \Delta \sigma_{S_{l}} \mathbb{1}_{\left(S_{l}, 1\right]}(t), \quad 0 \leqslant t \leqslant 1, \quad \Delta \sigma_{\infty} \stackrel{\text { def }}{=} 0
$$


and observe that $\psi$ is $\left(\mathcal{F}_{t}\right)$ adapted since $\sigma$ is $\left(\mathcal{F}_{t}\right)$ adapted and $\left(S_{l}\right)$ are stopping times as proven previously. Note that $\psi$ is simply $\sigma$ without jumps. Since $\psi$ is pathwise $\alpha$-Hölder continuous, we can define

$$
K(\omega)=\left(\sup _{\substack{0 \leq s<t \leq 1, s, t \in \mathbb{Q}}} \frac{\left|\psi_{t}(\omega)-\psi_{s}(\omega)\right|}{|t-s|^{\alpha}}\right) \vee \sup _{\substack{0 \leq t \leq 1, t \in \mathbb{Q}}}\left|\sigma_{t}(\omega)\right|<\infty, \quad \omega \in \Omega .
$$

$K$ is obviously measurable and fulfills the requirements of the Assumptions 2.4. Compare for similar results in this context also Chapter I, Proposition 1.32 in Jacod and Shiryaev [5].

Acknowledgments. The financial support of the Deutsche Forschungsgemeinschaft (FOR 916, project B4) is gratefully acknowledged.

\section{References}

[1] Aït-Sahalia, Y., Jacod, J. (2009) Testing for jumps in a discretely observed process. Ann. Statist. 37 (1), 184-222.

[2] Arnold, V. I. (2006) Ordinary Differential Equations. Springer Press. Translated from the 3th Russian original edition.

[3] Barndorff-Nielsen, O. E., Shephard, N. (2006). Econometrics of Testing for Jumps in Financial Economics Using Bipower Variation. Journal of Financial Econometrics 4, 1-30.

[4] HaAn, L., Ferreira, A. (2006). Extreme Value Theory: An Introduction. Springer Press.

[5] Jacod, J., Shiryaev, A. N. (2002). Limit theorems for stochastic processes, 2. Edition. Springer Press.

[6] Jacod, J., Todorov, V. (2010) Do price and volatility jump together? Ann. Appl. Probab. 20 (4), 1425-1469.

[7] Lee, S. S., Mykland, P. A. (2008). Jumps in Financial Markets: A New Nonparametric Test and Jump Dynamics. The Review of Financial Studies v 21 n 6, 2535-2563.

[8] Palmes, C. (2013). Statistical Analysis for Jumps in certain Semimartingale Models. (http://hdl.handle.net/2003/30367, Dissertation).

[9] Palmes, C., Woerner J.H.C. (2013). The Gumbel test for jumps in stochastic volatility models. (http://hdl.handle.net/2003/30625, submitted Preprint). 


\section{Preprints ab 2011/14}

2013-15

2013-14

2013-13

2013-12

2013-11

2013-10

2013-09

2013-08

2013-07

2013-06

2013-05

2013-04

2013-03

2013-02

2013-01

2012-19

2012-18

Christian Palmes and Jeannette H. C. Woerner

The Gumbel test and jumps in the volatility process

\section{Karl Friedrich Siburg, Katharina Stehling, Pavel A. Stoimenov,} Jeannette H. C. Woerner

An order for asymmetry in copulas, and implications for risk management

Michael Voit

Product formulas for a two-parameter family of Heckman-Opdam

hypergeometric functions of type $\mathrm{BC}$

\section{B. Schweizer and M. Veneroni}

Homogenization of plasticity equations with two-scale convergence methods

\section{Sven Glaser}

A law of large numbers for the power variation of fractional Lévy processes

Christian Palmes and Jeannette H.C. Woerner

The Gumbel test for jumps in stochastic volatility models

Agnes Lamacz, Stefan Neukamm, and Felix Otto

Moment bounds for the corrector in stochastic homogenization of a percolation model

Frank Klinker

Connections on Cahen-Wallach spaces

Andreas Rätz and Matthias Röger

Symmetry breaking in a bulk-surface reaction-diffusion model for signaling networks

Gilles Francfort and Ben Schweizer

A doubly non-linear system in small-strain visco-plasticity

\section{Tomáš Dohnal}

Traveling Solitary Waves in the Periodic Nonlinear Schrödinger Equation with Finite Band Potentials

Karl Friedrich Siburg, Pavel Stoimenov, and Gregor N.F. Weiß

Forecasting Portfolio-Value-at-Risk with Nonparametric Lower Tail Dependence Estimates

\section{Martin Heida}

On thermodynamics of fluid interfaces

\section{Martin Heida}

Existence of soulutions for two types of generalized versions

of the Cahn-Hilliard equation

\section{T. Dohnal, A. Lamacz, B. Schweizer}

Dispersive effective equations for waves in heterogeneous media on large time scales

\section{Martin Heida}

On gradient flows of nonconvex functionals in Hilbert spaces with

Riemannian metric and application to Cahn-Hilliard equations

R.V. Kohn, J. Lu, B. Schweizer, and M.I. Weinstein

A variational perspective on cloaking by anomalous localized resonance 
Olshanski spherical functions for infinite dimensional motion groups of fixed rank

$2012-16$

Selim Esedoḡlu, Andreas Rätz, Matthias Röger

Colliding Interfaces in Old and New Diffuse-interface Approximations of Willmore-flow

$2012-15$

Patrick Henning, Mario Ohlberger and Ben Schweizer

An adaptive multiscale finite element method

$2012-14$

$2012-13$

\section{Andreas Knauf, Frank Schulz, Karl Friedrich Siburg}

Positive topological entropy for multi-bump magnetic fields

Margit Rösler, Tom Koornwinder, and Michael Voit

Limit transition between hypergeometric functions of type BC and Type A

$2012-12$

Alexander Schnurr

Generalization of the Blumenthal-Getoor Index to the Class of Homogeneous

Diffusions with Jumps and some Applications

$2012-11$

$2012-10$

2012-09

2012-08

2012-07

2012-06

2012-05

2012-04

2012-03

2012-02

2012-01

$2011-14$

\section{Wilfried Hazod}

Remarks on pseudo stable laws on contractible groups

\section{Waldemar Grundmann}

Limit theorems for radial random walks on Euclidean spaces of high dimensions

\section{Martin Heida}

A two-scale model of two-phase flow in porous media ranging from porespace to the macro scale

\section{Martin Heida}

On the derivation of thermodynamically consistent boundary

conditions for the Cahn-Hilliard-Navier-Stokes system

Michael Voit

Uniform oscillatory behavior of spherical functions of $G L_{n} / U_{n}$ at the identity and a central limit theorem

\section{Agnes Lamacz and Ben Schweizer}

Effective Maxwell equations in a geometry with flat rings of arbitrary shape

Frank Klinker and Günter Skoruppa

Ein optimiertes Glättungsverfahren motiviert durch eine technische Fragestellung

Patrick Henning, Mario Ohlberger, and Ben Schweizer

Homogenization of the degenerate two-phase flow equations

Andreas Rätz

A new diffuse-interface model for step flow in epitaxial growth

Andreas Rätz and Ben Schweizer

Hysteresis models and gravity fingering in porous media

\section{Wilfried Hazod}

Intrinsic topologies on H-contraction groups with applications to semistability

Guy Bouchitté and Ben Schweizer

Plasmonic waves allow perfect transmission through sub-wavelength metallic gratings 